(1)

CrossMark

\title{
Revising our approach to conducting therapeutic clinical research in COPD
}

\author{
Gerard J. Criner \\ Affiliation: Dept of Thoracic Medicine and Surgery, Lewis Katz School of Medicine at Temple University, \\ Philadelphia, PA, USA. \\ Correspondence: Gerard J. Criner, Dept of Thoracic Medicine and Surgery, Lewis Katz School of Medicine at \\ Temple University, 745 Parkinson Pavilion, 3401 North Broad Street, Philadelphia, PA, USA. \\ E-mail: Gerard.Crinerdatuhs.temple.edu
}

@ERSpublications

Efficacy and effectiveness trials are needed to enable more effective treatment in COPD http://ow.ly/da2o30hPPNW

Cite this article as: Criner GJ. Revising our approach to conducting therapeutic clinical research in COPD. Eur Respir J 2018; 51: 1702712 [https://doi.org/10.1183/13993003.02712-2017].

Chronic obstructive pulmonary disease (COPD) is a major worldwide cause of death, and a leading cause of chronic disability and driver of high healthcare expenditures [1].

A great amount of research has been conducted over the past two decades by academia, industry and government supported research entities across the globe to transform COPD from a chronic disease that was previously considered largely untreatable, and hopelessly progressive, into a treatable condition [2]. However, despite these efforts, COPD continues to claim many lives and disable many more individuals without any cure in sight. There are many reasons for this, but some of the most important factors are that it is a chronic disorder hallmarked by frequent exacerbations of respiratory symptoms that occurs in a predominately older patient population who often have multiple comorbid conditions (heart disease, hypertension, obesity, diabetes, sleep abnormalities, musculoskeletal problems, lung cancer, etc.) that necessitate a complex care plan, especially in patients that are sicker [3]. These issues pose novel problems for clinicians who treat COPD patients in the community, older patients with protean pulmonary and non-pulmonary conditions who require a complex and comprehensive treatment plan but frequently lack significant financial or social resources to cover their medical expenses or help coach their medical care, respectively. What can clinical researchers do to change this paradigm and facilitate more effective treatments of COPD more rapidly into the patient community?

Randomised controlled trials (RCTs) have been considered the gold standard for determining the efficacy and safety of clinical treatments [4]. RCTs form the basis of most clinical guideline recommendations for patient care because of their accepted high scientific quality $[2,5]$. The conditions of a RCT maximise internal validity and the likelihood of detecting a treatment effect while simultaneously minimising harm. The study design accomplishes this goal by setting forth conditions that select an optimal patient population with strict oversight of outcome testing as well as monitoring for any adverse effects. RCT efficacy trials are geared to answer the question: "will an intervention provide clinically meaningful and statistically significant medical benefit without undue harm under optimal conditions?" This objective limits the eligible RCT patient participant pool to those without significant comorbid conditions, the extremes of age or airflow obstruction, those without current smoking or recent exacerbations, and those with the ability to read and write and comply with verbal and written instructions. Because most clinical

Received: Dec 282017 | Accepted: Jan 032018

Conflict of interest: None declared.

Copyright @ERS 2018 
research in COPD is conducted by academic centres in major urban centres, the RCT participant pool has the unintended bias of including individuals with higher educational levels, greater portability, greater financial and social support and, in some cases, includes a population of habitual study subjects due to clinical centre participant database mining. Many of the above factors may unintentionally select a patient population with COPD that may not reflect the profile of the general patient population that is treated at the community level.

Community clinical practice settings include patients who are at the extremes of age, with multiple comorbid conditions of varying severity and, potentially, problems with adherence to treatment plans or limited social and financial support. Moreover, the providers in those communities may have limited expertise in certain conditions and lack a comprehensive skill set to diagnose and gauge the severity of patients' problems related to COPD and institute effective disease specific treatment. It is apparent that there is a need to evaluate whether a treatment found to have efficacy in a RCT study is effective in routine clinical practice.

The paper of Woodcock et al. [6] in this issue of the European Respiratory Journal highlights the need for effectiveness research to complement efficacy research conducted within RCTs. They compared baseline characteristics, healthcare utilisation and outcomes of the Salford study, an open label effectiveness RCT, to six large scale efficacy RCTs that each included over 1000 subjects using inhaled therapy to assess its impact on exacerbation rates at 1 year. Not unexpectedly, the Salford study participants were older, had more females, more current smokers, more comorbidities and a greater number of prior exacerbations. During the study, Salford participants had higher rates of moderate and severe exacerbations, a higher incidence of serious adverse events and more severe episodes of pneumonia. Of note, only a maximum of $30 \%$ of patients enrolled in the Salford study would have been eligible for a phase III efficacy RCT and only $15 \%$ of Salford subjects would have been eligible for one of the six comparative RCTs.

Several aspects of the Salford study highlight some of the limitations in conducting effectiveness trials: less testing with spirometry was performed; more current smokers or subjects with a prior history of asthma were included; and patients were allowed to cross over to the usual care from the intervention arm during the study. A greater number of adverse events and serious adverse events and acute drug reactions were recorded. These factors limit our ability to understand if the patient population is truly comparable to efficacy based trials and whether the causes of the adverse events were related to the effects of the intervention, the presence of comorbid conditions, or underlying severity of COPD.

It is clear that chronic diseases like COPD that afflict a large number of patients with unique and diverse features are not reliably represented in RCTs, and this limits our ability to address important treatment needs and safety concerns in the broader patient population. The need for clinical or comparative effectiveness research to improve the delivery of clinical care into the community has been advocated by the Institute of Medicine and the Department of Health and Human Services, and led to the Affordable Care Act in the USA establishing the Patient-Centered Outcomes Research Institute to enable patient and community engagement to assist in the development of pertinent clinical trials [7-9]. Hopefully, combined efforts like these that incorporate patient and clinical community engagement with efficacy and effectiveness research will generate novel and transformative treatments more swiftly in all patients with COPD.

\section{References}

1 Global, regional, and national deaths, prevalence, disability-adjusted life years, and years lived with disability for chronic obstructive pulmonary disease and asthma, 1990-2015: a systematic analysis for the Global Burden of Disease Study 2015. Lancet Respir Med 2017; 5: 691-706.

2 Vogelmeier CF, Criner GJ, Martinez FJ, et al. Global Strategy for the Diagnosis, Management, and Prevention of Chronic Obstructive Lung Disease 2017 Report: GOLD Executive Summary. Eur Respir J 2017; 49: 1700214.

3 Cavailles A, Brinchault-Rabin G, Dixmier A, et al. Comorbidities of COPD. Eur Respir Rev 2013; 22: 454-475.

4 Bothwell LE, Podolsky SH. The emergence of the randomized, controlled trial. N Engl J Med 2016; 375: 501-504.

5 Carson SS, Goss CH, Patel SR, et al. An official American Thoracic Society research statement: comparative effectiveness research in pulmonary, critical care, and sleep medicine. Am J Respir Crit Care Med 2013; 188: $1253-1261$.

6 Woodcock A, Boucot I, Leather DA, et al. Effectiveness versus efficacy trials in COPD: how study design influences outcomes and applicability. Eur Respir J 2018; 51: 1701531.

7 Ellis LE, Kass NE. Patient engagement in patient-centered outcomes research: challenges, facilitators and actions to strengthen the field. J Comp Eff Res 2017; 6: 363-373.

8 Sox HC, Greenfield S. Comparative effectiveness research: a report from the Institute of Medicine. Ann Intern Med 2009; 151: 203-205.

9 Conway PH, Clancy C. Comparative-effectiveness research-implications of the Federal Coordinating Council's report. N Engl J Med 2009; 361: 328-330. 